\title{
The use of surgical sealants in the repair of dural tears during non-instrumented spinal surgery
}

\author{
Massimo Miscusi · Filippo Maria Polli · Stefano Forcato • \\ Maria Antoaneta Coman • Luca Ricciardi • \\ Alessandro Ramieri · Antonino Raco
}

Received: 24 February 2013/Revised: 12 December 2013/Accepted: 13 December 2013/Published online: 3 January 2014

(C) The Author(s) 2014. This article is published with open access at Springerlink.com

\begin{abstract}
Purpose To compare the success in repair of dural tears (DTs) using two different surgical sealants in non-instrumented lumbar spinal surgery and evaluate the incidence of associated short- and long-term complications.

Methods Twenty-three patients undergoing non-instrumented spinal surgery with intraoperative DTs were included both retrospectively and prospectively in this study. External signs of CSF leakage, neurological deficits, and infection-related complications were investigated postoperatively. The persistence of low-back pain was also evaluated and postoperative MRI was performed in all patients. DTs were repaired intraoperatively using suture with or without a dural patch. Eleven patients received an application of fibrin glue (Tissucol ${ }^{\circledR}$; Baxter, Inc., IL, USA) and 12 patients received an application of bovine serum albumin glutaraldehyde surgical adhesive (BioGlue ${ }^{\circledR}$ Surgical Adhesive; CryoLife, GA, USA). These patients were followed up at 3 months and 1 year postoperatively.

Results Successful intraoperative DT repair was obtained in all cases. Three patients in the Tissucol group presented with CSF leakage in the early postoperative period. There were no complications observed in the patients treated with BioGlue. At 3-month follow-up, no incidences of neurological or infection-related complications were observed in either group. There was no statistically significant difference in VAS between the two treatment groups.
\end{abstract}

M. Miscusi $(\bowtie) \cdot$ M. A. Coman · L. Ricciardi · A. Ramieri Department of Medico-Surgical Sciences Biotechnologies, Sapienza University of Rome, Latina, Italy

e-mail: m.miscusi@libero.it; massimo.miscusi@uniroma1.it

F. M. Polli · S. Forcato $\cdot$ A. Raco

Department of NESMOS, Sapienza University of Rome,

Rome, Italy
Conclusions Intraoperative DTs can be easily repaired by many effective techniques. However, in our experience, the use of BioGlue is an effective adjunct to immediate dural repair, being comparable in terms of efficacy and safety to the use of fibrin glue, potentially decreasing the incidence of associated short- and long-term complications.

Keywords BioGlue - CSF leakage - Dural tear . Duraplasty $\cdot$ Fibrin glue $\cdot$ Incidental durotomy

\section{Introduction}

Dural tear (DT) is the most frequent complication of spinal surgery $[1,2]$. DT rates in lumbar spinal surgery range from 3.1 to $17.4 \%$ [3-12]. Primary risk factors for DTs in lumbar surgery are reoperation, prior irradiation, or extensive degeneration of the lumbar spine [5]. These risk factors cause dural fibrosis or scarring that makes manipulation of the dural sac and root more difficult. Longer operative times, use of instrumentation, and a less experienced surgeon represent other significant risk factors for DT $[1,10]$.

DTs are inevitably associated with cerebrospinal fluid (CSF) leakage, causing subcutaneous CSF collections, postural headaches, and an increased risk of infectionrelated complications. DTs are frequently associated with radicular impairment due to direct root damage or the formation of a peri-radicular CSF pseudocyst and secondary spondylodiscitis $[1-4,9]$.

DTs are also associated with short- and long-term clinical sequelae, such as longer recuperative bed rest times, reoperations, extended inability to work, increased back pain, and a higher frequency of postoperative complications such as headaches [9]. Nevertheless, it has been 
clearly reported that when a DT is recognized and successfully repaired during surgery, it neither increases morbidity nor affects clinical outcome [7].

Various methods have been successfully used to repair DTs (suture, closed subarachnoid drainage, laser tissue welding, muscle, fat or fascial grafts, blood patches, fibrinadhesive sealant, gel foam, and bed rest). A treatment can be considered successful upon definitive repair of the DT and resolution of CSF leakage, with no subsequent neurological and infection-related complications. Intraoperatively, the Valsalva maneuver can be used to test the integrity of a DT repair, confirming a watertight seal has been achieved.

Moreover, a safe and effective treatment should not result in prolonged bed rest time, which may lead to complications such as deep vein thrombosis. This is of particular importance in cases of benign degenerative spinal disease, in which any complication or modification of the normal clinical course should be avoided or promptly resolved.

\section{Materials and methods}

Three hundred and twenty-one (321) patients undergoing non-instrumented lumbar spinal surgery procedures, including 218 microdiscectomy (MD) and 103 laminectomy (LM), in our department from January 2005 to August 2011 were included in this study. Data collected on patients before 2009 were obtained from the retrospective review of medical charts. After 2009, informed consent was obtained for all patients.

Of these 321 patients, 54 (16.8\%) were undergoing a reoperation (41 for recurrent herniated disc and 13 for repeat LM). Twenty-three patients $(7.1 \%)$ experienced DTs (13 patients undergoing MD and 10 undergoing LM). Fifteen $(65.2 \%)$ of the patients who experienced DTs were undergoing reoperations ( 9 patients undergoing MD and 6 undergoing LM). DTs involved radicular nerves in 12 cases, dural sac in 5 cases, and both in 6 cases.

In 16 cases of DT, primary repair was performed with 5-0 silk sutures; these cases included 5 lacerations of the dural sac, 10 of radicular nerve, and 1 case of both. In the remaining 7 cases (2 lacerations of axilla of radicular nerve and 5 of extremely fragile dura with involvement of sac and root dura), a small dural patch composed of equine collagen (Tissudura ${ }^{\circledR}$; Baxter, Inc.; IL, USA) was laid over the DT and partially sutured with 5-0 silk sutures. Two different materials were used to reinforce the sutures. In 11 cases, a thick layer of Tissucol ${ }^{\circledR}$ (Baxter, Inc.; IL, USA) fibrin glue was applied to suture alone $(n=9)$ or dural patch with suture $(n=2)$. Five patients had a radicular durotomy, 4 had a dural sac laceration, and 2 had both. Seven of these patients were undergoing reoperations. In the remaining 12 cases, a thin layer of BioGlue ${ }^{\circledR}$ Surgical Adhesive (BioGlue; CryoLife; GA, USA) was applied to suture alone $(n=7)$ or dural patch with suture $(n=5)$. Seven patients had a radicular durotomy, 1 had a dural sac laceration, and 4 had both. Eight of these patients were undergoing reoperations.

Factors contributing to the complexity of dural repair required are summarized in Table 1, grouped according to the sealant used. Seven out of the 11 patients in the Tissucol group (64\%) were reoperations, compared with 8 out of the 12 in the BioGlue group (67\%). Similarly, 4 out of 11 procedures in the Tissucol group (36\%) involved repairs associated with nerve laceration, compared with 6 out 11 in the BioGlue group (50\%). There were no significant differences in baseline characteristics between the two patient groups. Risk factors for CSF leaks, such as reoperations and the presence of nerve lacerations, were similar in both the Tissucol and BioGlue patients.

To test the integrity of the DT repairs and the absence of any intraoperative CSF leakage, the Valsalva maneuver was performed in triplicate for all cases.

After DT repair, the paravertebral fascial and subcutaneous muscles and skin layers were separately sutured. No drains were inserted in any of the cases.

For those patients who experienced DTs, our protocol prescribes that bed rest time is prolonged for at least $72 \mathrm{~h}$, compared to the standard $24 \mathrm{~h}$, in absence of further complications. During the immediate postoperative period, signs of external CSF leakage or subcutaneous collections were investigated. These patients underwent 3-month and 1-year clinical, imaging, and laboratory follow-up.

Table 1 Summary of patient characteristics

\begin{tabular}{|c|c|c|c|c|c|c|}
\hline Sealant & Number of patients & Type and number of procedures & Reoperations & Dural sac repair & Nerve laceration & Both procedures \\
\hline \multirow[t]{2}{*}{ Tissucol group } & \multirow[t]{2}{*}{11} & MD 7 & 4 & 1 & 2 & 1 \\
\hline & & LM 4 & 3 & 1 & 2 & 1 \\
\hline \multirow[t]{2}{*}{ BioGlue group } & \multirow[t]{2}{*}{12} & MD 6 & 5 & & 4 & 2 \\
\hline & & LM 6 & 3 & 1 & 2 & 3 \\
\hline \multirow[t]{2}{*}{ Total } & \multirow[t]{2}{*}{23} & MD 13 & 9 & 1 & 6 & 3 \\
\hline & & LM 10 & 6 & 1 & 4 & 4 \\
\hline
\end{tabular}




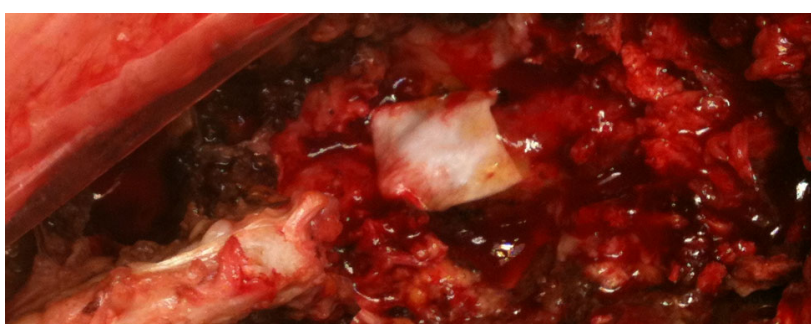

Fig. 1 Intraoperative photograph. A small dural patch composed of equine collagen (Tissudura ${ }^{\circledR}$; Baxter, Inc., IL, USA) was laid over the DT and a very thin layer of BioGlue ${ }^{\circledR}$ Surgical Adhesive (BioGlue; CryoLife, GA, USA) was applied to dural patch

The persistence of low-back pain was measured by Visual Analog Scale (VAS) scoring; signs of adjunctive postoperative neurological deficits and infection-related complications were evaluated; erythrocyte sedimentation rate (ESR) and C-reactive protein (CRP) were investigated. Magnetic resonance imaging (MRI) was performed to detect CSF leaks, pseudocysts, spinal arachnoiditis, or late spinal segment degeneration.

\section{Results}

Surgery and early postoperative period

In all 23 patients with DT, the dural defect was effectively repaired intraoperatively, indicated by the absence of CSF leakage. In two patients treated by dural patch, suture, and Tissucol, Valsalva maneuvers, resulted in sealant dislodgment, necessitating further reinforcement of the repair using a second, more effective application of Tissucol, before closing. In the group of 11 patients treated with Tissucol, three experienced CSF leakage in the early postoperative period. Two of them presented with external CSF leakage on the second and sixth postoperative days, respectively. These patients were initially treated with CSF lumbar drainage and prolonged bed rest for 1 week. One patient had resolution of CSF leakage; the other required a reoperation for repair of the dural defect with BioGlue. This surgical repair resulted in resolution of the CSF leakage. The third patient with CSF leakage presented with severe low-back pain on the tenth postoperative day owing to early spondylodiscitis due to Staphylococcus aureus. This patient required reoperation with debridement and irrigation of the surgical wound and received a muscle and fat patch sealed by Tissucol, which resulted in resolution of the CSF leak.

Another patient treated with Tissucol, who did not present with postoperative CSF leakage, experienced deep vein thrombosis in the early postoperative period, which was treated successfully with low molecular weight heparin for 3 months.

No signs of CSF leakage, either intra- or postoperatively, or neurological and infection-related complications were observed in any of the patients treated with BioGlue.

The mean bed rest time for patients with DT who experienced further complications (external CSF leakage, spondylodiscitis and deep venous thrombosis) was 15.2 days, and for patient with a successfully treated DT was 6.2 days. The standard bed rest time for patients who underwent non-instrumented lumbar surgery, without DT, was 1.2 days (Fig. 1).

Three-month follow-up

At 3-month follow-up, there were no cases of neurological complications observed in either group. Persistent low-back pain was reported in 5 patients with a mean VAS score of 45 (mean VAS score for all patients was 15); of these patients, 3 had been treated with Tissucol and 2 with BioGlue. In particular, the patient who experienced spondylodiscitis continued to present with severe low-back pain (VAS score of 65) without radicular involvement and was treated with antibiotics and a standard lumbosacral orthotic brace. In this patient, pain is most likely related to spondylodiscitis rather than duroplasty and sealant application. The patient who experienced deep vein thrombosis in the early postoperative period recovered and did not complain of low-back pain at 3-month follow-up. ESR and CRP were normal in all cases except for the patient with spondylodiscitis (ESR 25 and CRP 14).

\section{One-year follow-up}

At 1-year follow-up, 4 of the 5 patients who complained of low-back pain at 3-month follow-up, continued to present with discontinuous low-back pain, with a mean VAS score of 26 (mean VAS score for all patient was 10); of these patients, 2 had been treated with Tissucol and 2 with BioGlue. No patients demonstrated any neurological deficiencies. There was no statistically significant difference found in mean VAS scores between treatment groups $(p>0.1)$.

MRIs performed during follow-up confirmed that there were no cases of CSF leaks, pseudocysts or spinal arachnoiditis. No signs of late spinal segment degeneration were observed in either group, except for the patient with spondylodiscitis who underwent L4-L5 anterior fusion due to complete degeneration of the disc. No local or systemic adverse reactions to Tissucol or BioGlue were observed during the follow-up period (Fig. 2). 
Fig. 2 Axial T2 (left) and T1 (right) MRI scans at L4-L5 level. The dorsal left portion of dural sac has been repaired by a thin layer of BioGlue (arrows). No signs of extradural CSF collection are evident. The thin layer of BioGlue does not produce any compression or dislocation of dural sac or nerve root
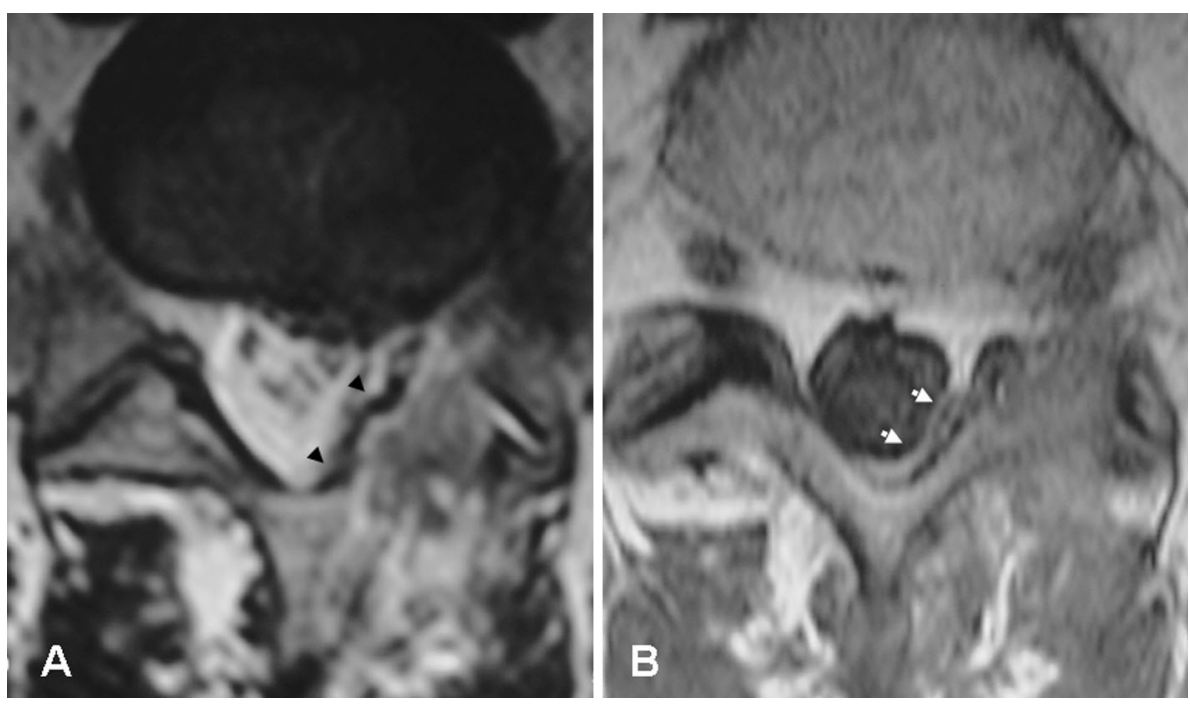

\section{Discussion}

DT is the most frequent complication of spinal surgery, and has a higher tendency of occurring during dissection of the epidural scar at reoperation $[13,14]$. We believe that DTs should be treated immediately and effectively to avoid further complications.

Surgeons tend to have personal preferences for the effective treatment of DTs, and the literature reports many safe and long-standing surgical strategies as follows: sutures; closed subarachnoid drainage; prolonged subfascial drain placement; laser tissue welding; muscle, fat or fascial grafts; blood patches; fibrin adhesive or cyanoacrylate polymer sealants; gel foam; autologous fibrin tissue adhesive; polyglycolic acid mesh with fibrin glue; and absorbable hydrogels such as DuraSeal ${ }^{\mathrm{TM}}$ [3, 13-18].

Surgical factors that could impede detection, successful repair, and healing of DTs should be avoided. For example, ADCON-L ${ }^{\circledR}$ (Gliatech; OH, USA), a porcine-derived polyglycan used in spinal surgery to reduce postoperative epidural fibrosis and adhesion, may inhibit dural healing and exacerbate CSF leakage from microscopic durotomies that may be undetectable at the time of surgery [19].

Suturing the DT whenever possible appears to be an important factor in the prevention of repeat surgery to repair the defect [8]. Reoperation for dural repair is recommended when CSF leakage is noted in the early postoperative period and is mandatory when the CSF leakage does not resolve spontaneously or by lumbar drainage, or when it is associated with signs of wound infection [20]. Immediate and effective repair of DT is particularly important also because DT is reported to be the second most frequent cause of medicolegal implications after nerve root damage [21].
Our treatment protocol includes, as a first priority, the watertight suture of the DT, if possible. Nevertheless, there are some instances when suturing is technically difficult, as in root axillary durotomies or in patients with fragile and very thin or scarred dural layer. In such cases, we suggest the partial suturing of a small dural patch to avoid any further dural damage, followed by an application of a dural sealant. The dural patch may be synthetic or biologic; in the latter case, heterologous (bovine or equine) or autologous (muscle or fat) may be used.

At our institution, we utilize Tissucol and BioGlue as dural sealants. They have different characteristics and hence should be used in different manners. Tissucol is a topical hemostat, composed of fibrinogen, aprotinin, human thrombin, and calcium chloride. When Tissucol is applied, a dense fibrin network is formed which facilitates DT repair via its hemostatic properties. In contrast, BioGlue forms covalent bonds with cell surface proteins once in contact with the tissue. Because of the difference between Tissucol and BioGlue in mechanism of action, a thin layer of BioGlue is sufficient to achieve repair of the DT without complications. In this patient series, Valsalva maneuvers were performed before closure.

CSF leaks were observed at the repair sites in two patients where Tissucol had been applied but had apparently been dislodged. Reapplication of the sealant subsequently achieved full duroplasty. No CSF leaks were observed following Valsalva maneuvers where BioGlue was used for DT repair.

Although there were no significant differences in the dural defects of the two groups, we speculate that DT repairs with the use of BioGlue, due to its strong bonding properties, make it more resistant soon after the application to internal CSF pressure and pulses which could consequently force the suture open, dislodge the applied sealant, 
and reopen the leak. Therefore, for a faster and more confident technique for controlling CSF leakage during surgery, we have moved increasingly toward the use of BioGlue for DT repair at our institution.

Even in cases of effective treatment of DTs, as reported in the literature, it was our common practice to prolong the postoperative bed rest time, leaving the patient supine for a few days [3, 22]. In this series, patients with complications from DT and postoperative CSF leakage had a longer bed rest time as compared to patients with successfully repaired DT.

In our experience, a single, very thin layer of BioGlue is sufficient to reinforce the dural repair site and to prevent any secondary ruptures; it is also effective in assuring good adhesion of the edges of the dural patch to the native surrounding dura [23]. Furthermore, the gradual resorption of BioGlue allows for sufficient time for local healing of the DT [24]. Nevertheless, it is essential that a very thin layer of BioGlue is applied; the application of larger quantities may create a pooling of glue, potentially leading to serious side effects, such as compression of the spinal cord and nerve roots [25]. If used incorrectly, excess glue may isolate a dead space at the surgical site from blood and antibiotics, thus facilitating infections [26].

Toxic effects of BioGlue have been anecdotally reported in the literature; these effects have been associated with the possible development of aseptic meningitis due to the glutaraldehyde component [23, 27]. However, it should be noted that the bovine serum albumin $(45 \%)$ and glutaraldehyde $(10 \%)$ are mixed together in the applicator tip to form the glue, after which point the glutaraldehyde no longer exists as a single component. Although the biocompatibility of this sealant has been demonstrated, care should be taken to apply it only extradurally [28].

BioGlue has been used extensively in neurosurgery, in both cranial and spinal procedures [29]. It has been used with particular success in preventing postoperative CSF leaks in transsphenoidal surgery [30, 31]. In all our patients, in both cranial and spinal surgery, we have never experienced complications directly related to its use. Also, since this is a retrospective study, a controlled comparison of Tissucol to BioGlue in the repair of DT was not possible. This observational study shows that BioGlue use was effective in intraoperative DT repair and did not result in any complications. BioGlue has also been very useful in select and challenging cases such as reoperation in the posterior fossa, where a scarred and fibrotic dura can lose its physiological elasticity and resilience, being exposed to the mechanical stress and bulging of CSF pulses [32].

\section{Conclusion}

BioGlue and Tissucol are effective and safe dural sealants, allowing for immediate dural repair in non-instrumental lumbar spinal surgery. When used appropriately and in an informed manner, BioGlue can potentially reduce the incidence of associated short- and long-term complications. Further studies to evaluate the effects of BioGlue use on the incidence of complications associated with the repair of dural tears are warranted.

Conflict of interest Miscusi Massimo $\mathrm{PhD}$, MD was scientific consultant for Eurolife Europe LTD from 2008 to 2010.

Open Access This article is distributed under the terms of the Creative Commons Attribution License which permits any use, distribution, and reproduction in any medium, provided the original author(s) and the source are credited.

\section{References}

1. Bosacco SJ, Gardner MJ, Guille JT (2001) Evaluation and treatment of dural tears in lumbar spine surgery: a review. Clin Orthop Relat Res 389:238-247

2. Tafazal SI, Sell PJ (2005) Incidental durotomy in lumbar spine surgery: incidence and management. Eur Spine J 14:287-290

3. Cammisa FP Jr, Girardi FP, Sangani PK, Parvataneni HK, Cadag S, Sandhu HS (2000) Incidental durotomy in spine surgery. Spine 25:2663-2667

4. Carmouche JJ, Molinari RW (2004) Epidural abscess and discitis complicating instrumented posterior lumbar interbody fusion: a case report. Spine 29:E542-E546

5. Epstein NE (2007) The frequency and etiology of intraoperative dural tears in 110 predominantly geriatric patients undergoing multilevel laminectomy with noninstrumented fusions. J Spinal Disord Tech 20:380-386

6. Fink LH (1996) Unintended "incidental" durotomy. Surg Neurol 45:590

7. Jones AA, Stambough JL, Balderston RA, Rothman RH, Booth RE Jr (1989) Long-term results of lumbar spine surgery complicated by unintended incidental durotomy. Spine 14:443-446

8. Khan MH, Rihn J, Steele G, Davis R, Donaldson WF 3rd, Kang JD et al (2006) Postoperative management protocol for incidental dural tears during degenerative lumbar spine surgery: a review of 3,183 consecutive degenerative lumbar cases. Spine 31:26092613

9. Saxler G, Krämer J, Barden B, Kurt A, Pforter J, Bernsmann K (2005) The long-term clinical sequelae of incidental durotomy in lumbar disc surgery. Spine 30:2298-2302

10. Sin AH, Caldito G, Smith D, Rashidi M, Willis B, Nanda A (2006) Predictive factors for dural tear and cerebrospinal fluid leakage in patients undergoing lumbar surgery. J Neurosurg Spine 5:224-227

11. Stolke D, Sollmann WP, Seifert V (1989) Intra- and postoperative complications in lumbar disc surgery. Spine 14:56-59

12. Wang JC, Bohlman HH, Riew KD (1998) Dural tears secondary to operations on the lumbar spine. Management and results after a two-year-minimum follow-up of eighty-eight patients. J Bone Joint Surg Am 80:1728-1732 
13. Black P (2002) Cerebrospinal fluid leaks following spinal surgery: use of fat grafts for prevention and repair. Technical note. J Neurosurg 96(Suppl 2):S250-S252

14. Boogaarts JD, Grotenhuis JA, Bartels RH, Beems T (2005) Use of a novel absorbable hydrogel for augmentation of dural repair: results of a preliminary clinical study. Neurosurgery 57(Suppl 1):S146-S151 (discussion S146-S151)

15. Hawk MW, Kim KD (2000) Review of spinal pseudomeningoceles and cerebrospinal fluid fistulas. Neurosurg Focus 9:E5

16. Hida K, Yamaguchi S, Seki T, Yano S, Akino M, Terasaka S et al (2006) Nonsuture dural repair using polyglycolic acid mesh and fibrin glue: clinical application to spinal surgery. Surg Neurol 65:136-142 (discussion 142-143)

17. Hughes SA, Ozgur BM, German M, Taylor WR (2006) Prolonged Jackson-Pratt drainage in the management of lumbar cerebrospinal fluid leaks. Surg Neurol 65:410-414

18. Nakamura H, Matsuyama Y, Yoshihara H, Sakai Y, Katayama Y, Nakashima $S$ et al (2005) The effect of autologous fibrin tissue adhesive on postoperative cerebrospinal fluid leak in spinal cord surgery: a randomized controlled trial. Spine 30:E347-E351

19. Le AX, Rogers DE, Dawson EG, Kropf MA, De Grange DA, Delamarter RB (2001) Unrecognized durotomy after lumbar discectomy: a report of four cases associated with the use of ADCON-L. Spine 26:115-117

20. Eismont FJ, Wiesel SW, Rothman RH (1981) Treatment of dural tears associated with spinal surgery. J Bone Joint Surg Am 63:1132-1136

21. Goodkin R, Laska LL (1995) Unintended "incidental" durotomy during surgery of the lumbar spine: medicolegal implications. Surg Neurol 43:4-12 (discussion 12-14)

22. Hodges SD, Humphreys SC, Eck JC, Covington LA (1999) Management of incidental durotomy without mandatory bed rest. A retrospective review of 20 cases. Spine 24:2062-2064
23. Miscusi M (2011) BioGlue and spine surgery. Spine J 11:983

24. Yuen T, Kaye AH (2005) Persistence of Bioglue in spinal dural repair. J Clin Neurosci 12:100-101

25. Woo EJ (2012) Dural repair with spinal sealants. Spine J 12:279

26. Klimo P Jr, Khalil A, Slotkin JR, Smith ER, Scott RM, Goumnerova LC (2007) Wound complications associated with the use of bovine serum albumin-glutaraldehyde surgical adhesive in pediatric patients. Neurosurgery 60(Suppl 2):S305-S309 (discussion 309)

27. Fürst W, Banerjee A (2005) Release of glutaraldehyde from an albumin-glutaraldehyde tissue adhesive causes significant in vitro and in vivo toxicity. Ann Thorac Surg 79:1522-1528 (discussion 1529)

28. Stylli SS, Kumar A, Gonzales M, Kaye AH (2004) The biocompatibility of BioGlue with the cerebral cortex: a pilot study. J Clin Neurosci 11:631-635

29. Kumar A, Maartens NF, Kaye AH (2003) Evaluation of the use of BioGlue ${ }^{\circledR}$ in neurosurgical procedures. J Clin Neurosci 10:661-664

30. Dusick JR, Mattozo CA, Esposito F, Kelly DF (2006) BioGlue for prevention of postoperative cerebrospinal fluid leaks in transsphenoidal surgery: a case series. Surg Neurol 66:371-376 discussion 376

31. Sen A, Green KM, Khan MI, Saeed SR, Ramsden RT (2006) Cerebrospinal fluid leak rate after the use of BioGlue in translabyrinthine vestibular schwannoma surgery: a prospective study. Otol Neurotol 27:102-105

32. Miscusi M (2004) Initial experience of a new surgical adhesive (BioGlue) in neurosurgery. In: XIX biennial Congress of the European Society of Pediatric Neurosurgery. Rome 6-9 May 2004 